\title{
Skeletal and dentoalveolar changes after miniscrew- assisted rapid palatal expansion in young adults: A cone-beam computed tomography study
}

\author{
Jung Jin Park \\ Young-Chel Park ${ }^{\mathrm{a}}$ \\ Kee-Joon Lee ${ }^{a}$ \\ Jung-Yul Cha ${ }^{\mathrm{a}}$ \\ Ji Hyun Tahk ${ }^{\mathrm{b}}$ \\ Yoon Jeong Choi ${ }^{\mathrm{a}}$
}

${ }^{a}$ Department of Orthodontics, Institute of Craniofacial Deformity, College of Dentistry, Yonsei University, Seoul, Korea

${ }^{\mathrm{b}}$ Graduate of Harvard School of Dental Medicine, Harvard University, Boston, MA, USA
Objective: The aim of this study was to evaluate the skeletal and dentoalveolar changes after miniscrew-assisted rapid palatal expansion (MARPE) in young adults by cone-beam computed tomography (CBCT). Methods: This retrospective study included 14 patients (mean age, 20.1 years; range, 16-26 years) with maxillary transverse deficiency treated with MARPE. Skeletal and dentoalveolar changes were evaluated using CBCT images acquired before and after expansion. Statistical analyses were performed using paired $t$-test or Wilcoxon signed-rank test according to normality of the data. Results: The midpalatal suture was separated, and the maxilla exhibited statistically significant lateral movement ( $p$ $<0.05$ ) after MARPE. Some of the landmarks had shifted forwards or upwards by a clinically irrelevant distance of less than $1 \mathrm{~mm}$. The amount of expansion decreased in the superior direction, with values of 5.5, 3.2, 2.0, and $0.8 \mathrm{~mm}$ at the crown, cementoenamel junction, maxillary basal bone, and zygomatic arch levels, respectively $(p<0.05)$. The buccal bone thickness and height of the alveolar crest had decreased by 0.6-1.1 $\mathrm{mm}$ and $1.7-2.2 \mathrm{~mm}$, respectively, with the premolars and molars exhibiting buccal tipping of $1.1^{\circ}-2.9^{\circ}$. Conclusions: Our results indicate that MARPE is an effective method for the correction of maxillary transverse deficiency without surgery in young adults.

[Korean J Orthod 2017;47(2):77-86]

Key words: Cone-beam computed tomography, Miniscrew-assisted rapid palatal expansion, Adults, Expansion

Received May 11, 2016; Revised July 6, 2016; Accepted July 13, 2016.

Corresponding author: Yoon Jeong Choi.

Assistant Professor, Department of Orthodontics, College of Dentistry, Yonsei University, 50-1 Yonsei-ro, Seodaemun-gu, Seoul 03722, Korea.

Tel +82-2-2228-3101 e-mail yoonjchoi@yuhs.ac

*This study was supported by a faculty research grant of Yonsei University College of Dentistry for 6-2015-0123.

The authors report no commercial, proprietary, or financial interest in the products or companies described in this article.

(C) 2017 The Korean Association of Orthodontists.

This is an Open Access article distributed under the terms of the Creative Commons Attribution Non-Commercial License (http://creativecommons.org/licenses/by-nc/4.0) which permits unrestricted non-commercial use, distribution, and reproduction in any medium, provided the original work is properly cited. 


\section{INTRODUCTION}

Rapid palatal expansion (RPE) has been widely used in the field of orthodontics since the mid-1960s for increasing the transverse dimensions of the maxilla in growing patients.' Rapid palatal expansion enables the separation of the midpalatal suture, which is followed by skeletal orthopedic expansion. ${ }^{2}$ Surgically assisted RPE (SARPE) is a treatment modality that helps overcome increased resistance from the bony palate and zygomatic buttress in adults. ${ }^{3,4}$ However, SARPE has several limitations, including high cost, a complex treatment process, and surgical morbidity, ${ }^{5}$ and most patients are reluctant to undergo this surgical procedure. Therefore, several efforts have been made to minimize the surgical risks and limitations of RPE.

Previous histological studies have shown that the midpalatal suture begins to obliterate during the juvenile stage, with a marked degree of closure observed in the third decade of life. ${ }^{6,7}$ Therefore, conventional RPE can produce unwanted effects in adults, such as expansion failure, alveolar bone dehiscence, buccal crown tipping, root resorption, reduction in buccal bone thickness, and marginal bone loss. ${ }^{8}$ To minimize these side effects, orthopedic expansion of the basal bone is essential in non-growing patients. ${ }^{9,10}$

Nonsurgical maxillary expansion can be achieved through conventional, bone-anchored, or combinationtype RPE. Bone-anchored devices have been reported to successfully expand the maxilla after lateral osteotomy. ${ }^{4,11}$ To ensure expansion of the basal bone without surgical intervention and maintain the separated bone in consolidation, Lee et al. ${ }^{12}$ introduced miniscrew-assisted RPE (MARPE) and reported successful expansion of the maxilla through opening of the midpalatal suture.

Periapical or occlusal radiographs are adequate for assessing the opening of the midpalatal suture. However, the movements of each tooth and its alveolus are barely identifiable on conventional two-dimensional (2D) radiographs such as lateral or posteroanterior (PA) cephalograms. Cone-beam computed tomography (CBCT) allows imaging at relatively low radiation dosages and presents a clear view of bony structures, with minimal image distortion. ${ }^{13}$ Skeletal and dentoalveolar changes after conventional tooth-borne and toothbone-borne RPE have been investigated using CBCT in growing patients. ${ }^{14-16}$ In contrast, there is limited information regarding nonsurgical expansion using boneborne techniques such as MARPE in young adults. Therefore, СВСТ can be used to accurately assess not only the changes in each tooth and its alveolus, but also quantitative three-dimensional (3D) changes in the maxillofacial complex after MARPE. ${ }^{17}$

The aim of the present study was to evaluate the following null hypothesis: short-term skeletal and dentoalveolar measurements obtained before and after MARPE in young adults do not differ significantly. To test the hypothesis, CBCT data acquired before and after MARPE were compared.

\section{MATERIALS AND METHODS}

\section{Subjects}

The CBCT records of 19 patients with maxillary constriction who had undergone maxillary expansion by MARPE between January 2012 and October 2013 and had a complete set of CBCT images acquired before (T1) and after (T2) expansion were retrieved from the archives of the Department of Orthodontics, Yonsei University Dental Hospital. Maxillary constriction was diagnosed at maxillomandibular transverse differential index values $>19.6 \mathrm{~mm}^{18}$ Of the 19 patients, 5 were excluded on the basis of the following exclusion criteria: failure of opening of the midpalatal suture $(\mathrm{n}=3)$, systemic diseases, craniofacial anomalies $(\mathrm{n}=1)$, and history of orthodontic treatment $(n=1)$. Finally, 14 patients (male, 9; female, 5) with a mean age of $20.1 \pm 2.4$ years (range, 16-26 years) were retrospectively enrolled in the study. None of the subjects exhibited functional displacement. The mean duration of expansion was 27 days (range, 18-35 days), and the mean amount of expansion was $6.7 \mathrm{~mm}$ (range, $4.5-8.8 \mathrm{~mm}$ ). The second set of CBCT images were acquired within 5 weeks (mean duration, 10.7 days; range, 1-35 days) of completion of expansion. The mean duration between $\mathrm{T} 1$ and $\mathrm{T} 2$ was 38 days (range, 24-66 days). This study was approved by the institutional review board of Yonsei University Dental Hospital (No. 2-2015-0017). Because of the retrospective nature of the study, the institutional review board waived the requirement for written informed consent from patients.

The MARPE device was fabricated by modifying the conventional hyrax-type RPE device. ${ }^{12}$ Four rigid connectors, composed of $0.8-\mathrm{mm}$ stainless steel wire with helical hooks, were soldered onto the base of the conventional hyrax screw body (Hyrax ${ }^{\circledR}$ Click; Dentaurum, lspringen, Germany). Two anterior hooks were positioned in the rugae area, and two posterior hooks were positioned in the para-midsagittal area. The MARPE device made passive contact with the underlying tissue. Following cementation of the appliance to the maxillary first premolars and molars, four orthodontic miniscrews (Orlus; Ortholution, Seoul, Korea), with a collar diameter of $1.8 \mathrm{~mm}$ and length of $7 \mathrm{~mm}$, were placed at the center of each helical hook (Figure 1). ${ }^{12}$ Maxillary expansion was initiated on the day after MARPE device placement. The appliance was activated at a rate of one turn per day ( $0.2 \mathrm{~mm}$ per turn) until the 
required expansion was achieved.

\section{Measurements}

The CBCT device (Alphard VEGA; ASAHI Roentgen IND, Kyoto, Japan) was set at $5.0 \mathrm{~mA}$ and $80 \mathrm{kV}$, and images were acquired for 17 seconds, with a voxel size of $0.3 \mathrm{~mm}$. During image acquisition, the patients were seated upright, with the Frankfort horizontal plane parallel to the floor and the patient's head stabilized by an ear rod. Patients were asked to open their mouth slightly during image acquisition to prevent overlapping

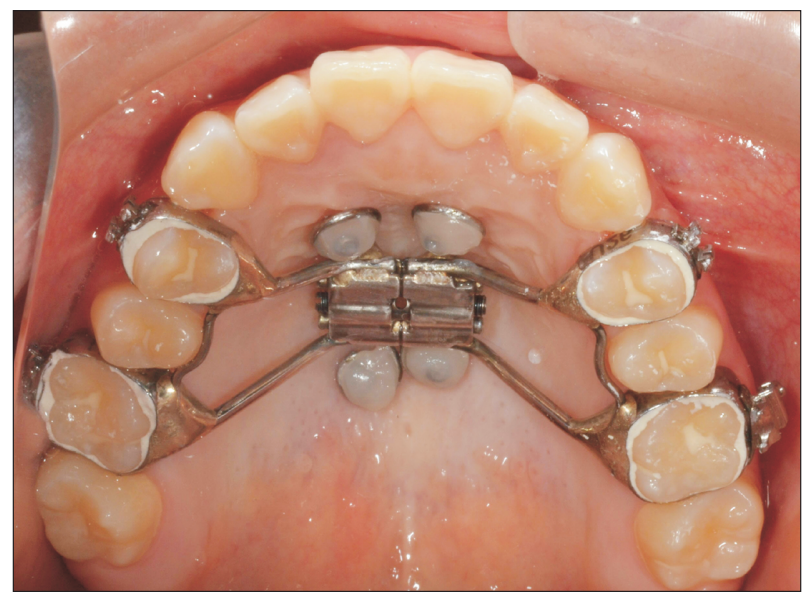

Figure 1. Clinical application of miniscrew-assisted rapid palatal expansion. of the maxillary and mandibular teeth. The images were imported as digital imaging and communications in medicine (DICOM) files using $\operatorname{lnVivo} 5^{\circledR}$ software (Anatomage, San Jose, CA, USA) and reoriented with the palatal plane parallel to the floor in the sagittal and coronal planes. Subsequent measurements were performed using the same software $\left(\operatorname{lnVivo} 5^{\circledR}\right)$.

Interpremolar (IPMW) and intermolar (IMW) widths,

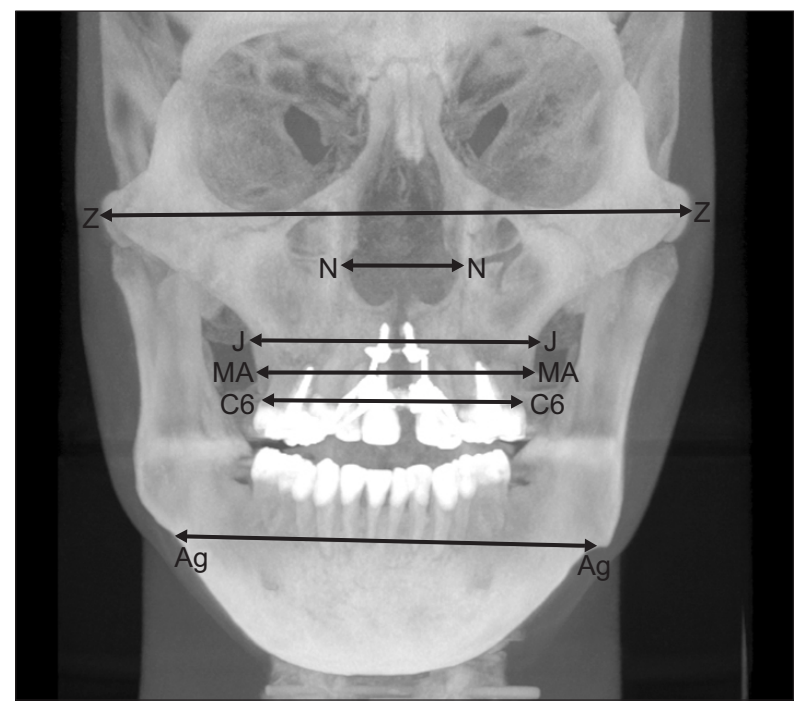

Figure 3. Two-dimensional posteroanterior cephalogram reconstructed from a three-dimensional skull model. Refer to Table 1 for the definitions of abbreviations.

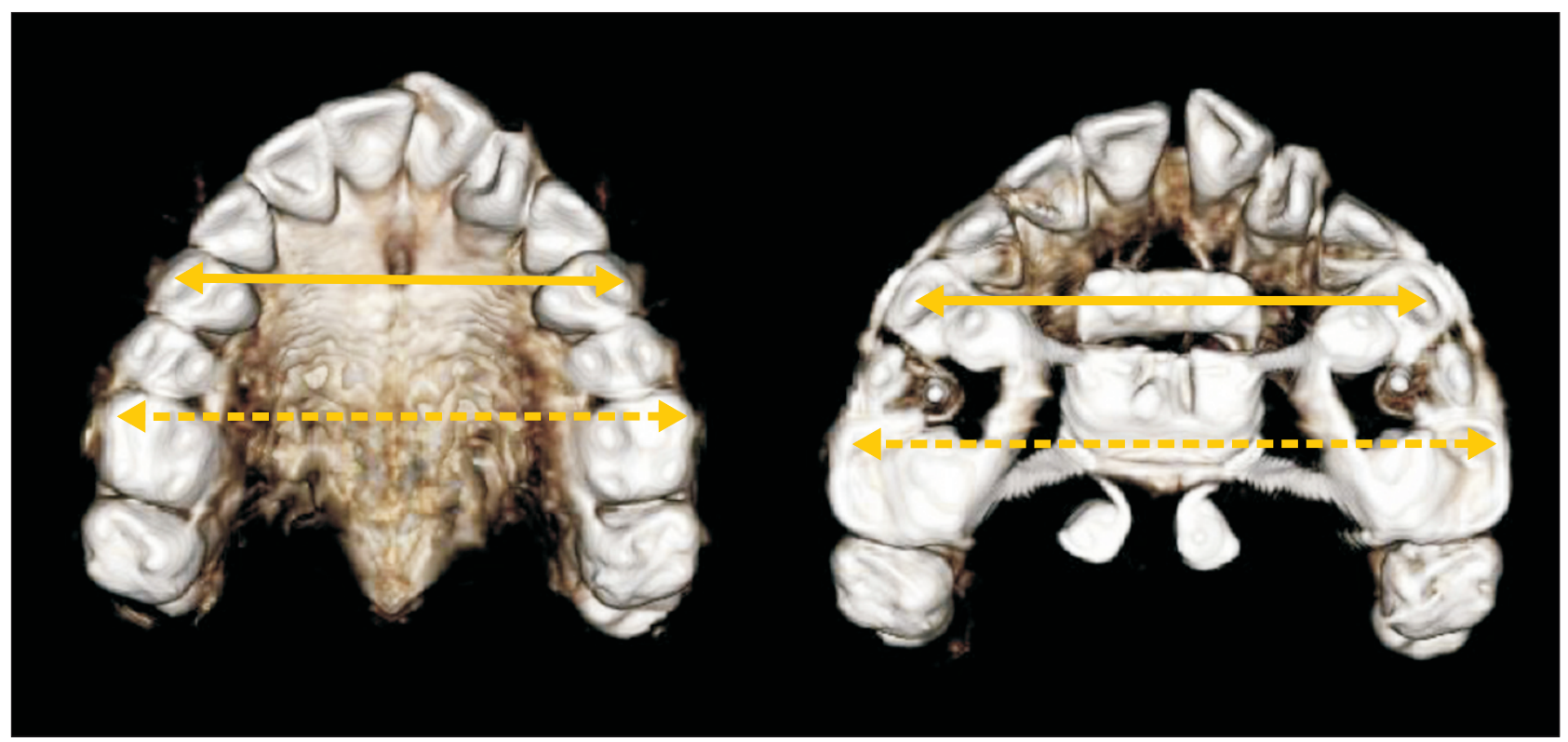

Figure 2. Three-dimensional tooth models used for the cone-beam computed tomography assessment of interpremolar and intermolar widths after miniscrew-assisted rapid palatal expansion.

Solid arrow, interpremolar width; dashed arrow, intermolar width. 
defined as the distances between the right and left buccal/mesiobuccal cusp tips of the first premolars and first molars, respectively, were measured on 3D tooth images (Figure 2). For measurement of transverse dimensions, 2D PA cephalograms were reconstructed perpendicular to the midsagittal plane. Bilateral landmarks ( $\mathrm{Z}, \mathrm{N}, \mathrm{J}, \mathrm{MA}, \mathrm{C} 6$, and $\mathrm{Ag})$ were identified in these images, and the distances between right and left corresponding landmarks were measured (Figure 3). The landmarks evaluated in this study and their definitions are summarized in Table 1.

Three-dimensional skull images acquired at $\mathrm{T} 1$

Table 1. Definition of landmarks used in this study

\begin{tabular}{ll}
\hline \multicolumn{1}{c}{ Landmark } & Description \\
\hline $\mathrm{Z}$ & The most lateral point of the zygomatic arch \\
$\mathrm{N}$ & The most lateral wall of the nasal cavity \\
$\mathrm{J}$ & Midpoint of the J and C6 points on the lateral contour of the maxillary alveolus \\
$\mathrm{MA}$ & The most lateral point of cemento-enamel junction of the maxillary first molar \\
$\mathrm{C} 6$ & Antegonial notch \\
Ag & The most infero-lateral point of the nasal aperture in a transverse plane \\
Alare & The most infero-lateral point on the alveolar ridge opposite the center of the maxillary canine \\
Ectocanine & The most posterior and deepest point on the anterior contour of the maxillary alveolar process in \\
A-point* & the mid-sagittal plane \\
& The most antero-inferior point on the maxillary alveolar margin in the mid-sagittal plane \\
Prosthion* & The most infero-lateral point on the alveolar ridge opposite the center of the maxillary first molar \\
Ectomolare & The most infero-lateral point of the processus zygomaticus \\
Processus zygomaticus &
\end{tabular}

Z, N, J, MA, C6, and Ag were identified on the reconstructed two-dimensional posteroanterior cephalogram of a threedimensional skull model.

Alare, ectocanine, A-point, prosthion, ectomolare, and processus zygomaticus were defined according to the study by Magnusson et al. ${ }^{19}$

*Although A-point and prosthion were one-point landmarks before expansion, they were separated into right and left landmarks after expansion.
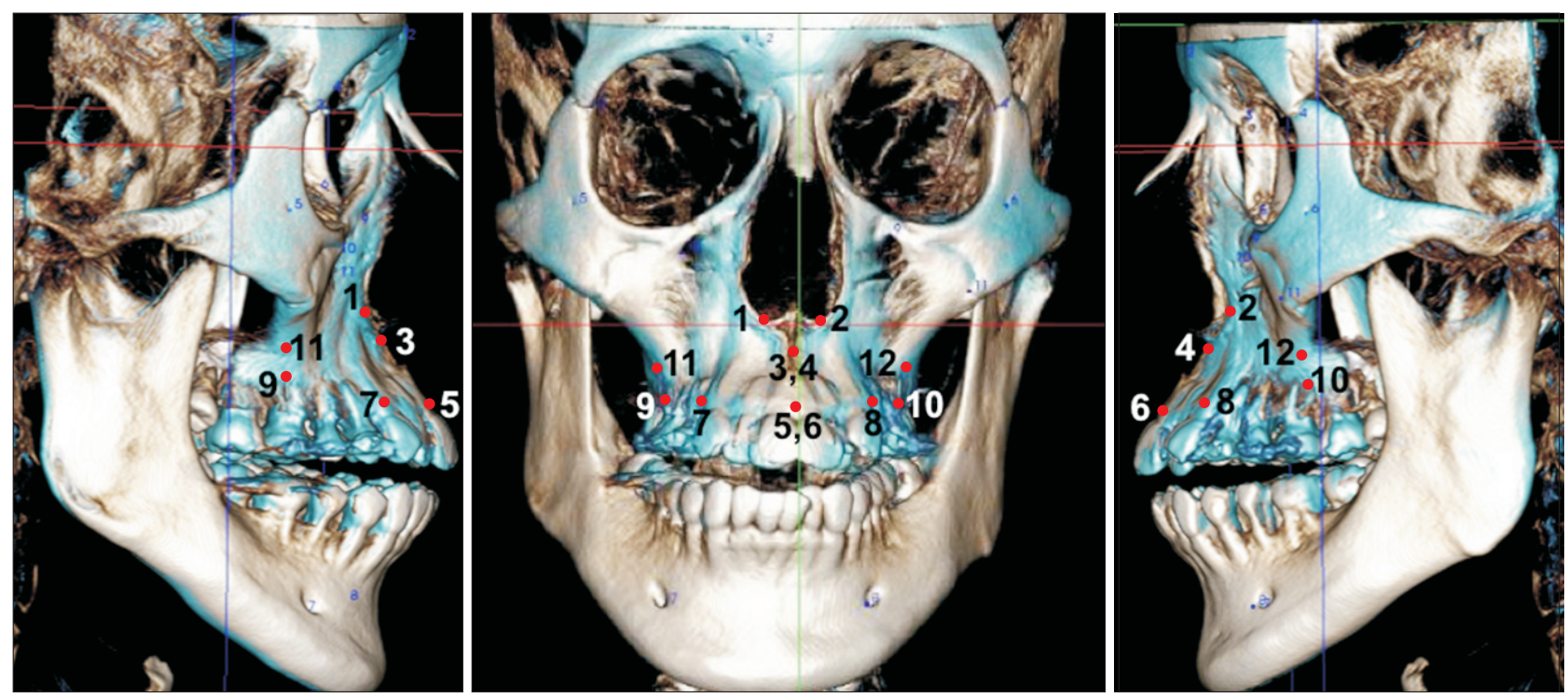

Figure 4. Superimposition of three-dimensional cone-beam computed tomography images acquired before (white) and after (blue) miniscrew-assisted rapid palatal expansion.

1 and 2, alare, right and left; 3 and 4, A-point, right and left; 5 and 6, prosthion, right and left; 7 and 8, ectocanine, right and left; 9 and 10, ectomolare, right and left; 11 and 12, processus zygomaticus, right and left. 

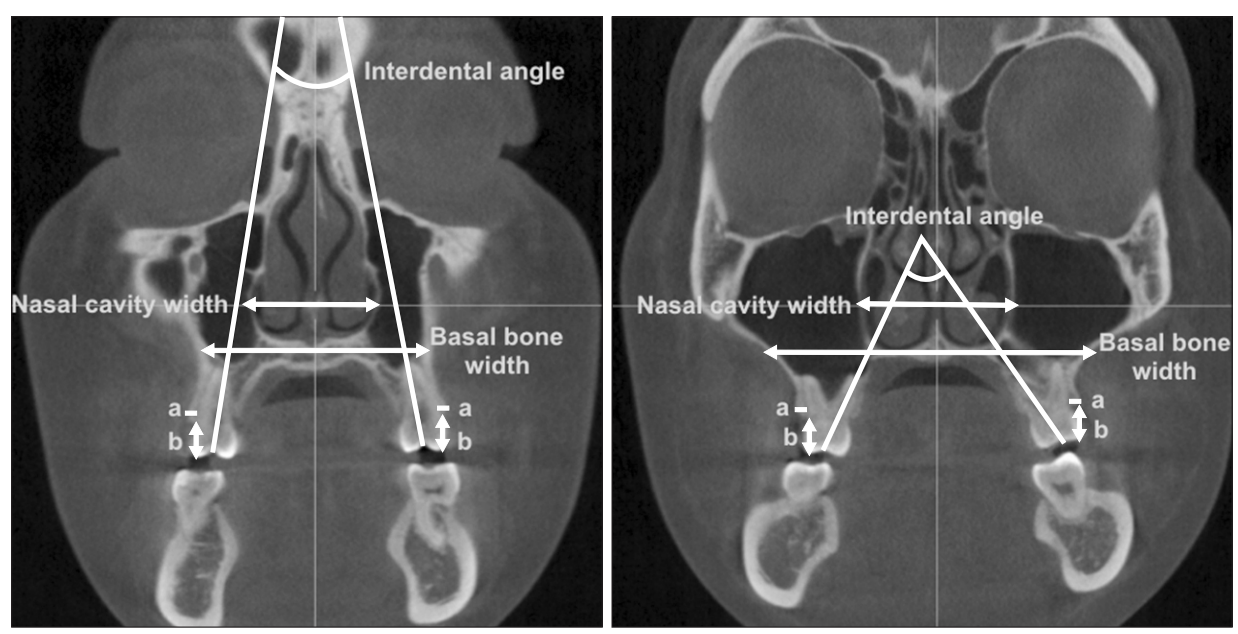

Figure 5. Coronal cone-beam computed tomography images acquired before expansion at furcations of the first premolar (left) and first molar (right).

$a$, buccal bone thickness; $b$, buccal alveolar height.

Buccal bone thickness and alveolar height were measured on the right and left sides, and the mean value of the two measurements was calculated.

and T2 were superimposed using a point-to-point and volumetric registration method with specifically normalized mutual data based on the anterior cranial base (Figure 4). ${ }^{19}$ Upon volumetric registration, the $\mathrm{T} 1$ and $\mathrm{T} 2$ images shared the same coordinate system, which compensated for discrepancies and minimized the risk of measurement errors. On each image, six pairs of bony landmarks were identified on the basis of a previous report, ${ }^{19}$ following which, each landmark was coordinated. Displacements in the maxilla were analyzed along the $\mathrm{x}, \mathrm{y}$, and $\mathrm{z}$ axes by calculating the deviation of each landmark between $\mathrm{T} 1$ and $\mathrm{T} 2$. The distances between right and left corresponding landmarks were measured, and the differences between the measurements in $\mathrm{T} 1$ and $\mathrm{T} 2$ were calculated.

Two coronal scans were obtained perpendicular to the midsagittal plane, passing through the buccal/ mesiobuccal cusp tips and furcations of the maxillary first premolars and molars. For the maxillary first premolars with a single root, coronal scans were obtained perpendicular to the midsagittal plane, passing through the buccal and palatal cusp tips. For measurement of nasal cavity and basal bone widths, the anterior-most slice showing the entire palatal roots of the maxillary right first premolars and molars was selected (Figure 5). On each image, the following parameters were measured: nasal cavity width, defined as the transverse width between the lateral-most points of each nasal cavity; basal bone width, defined as the transverse width between the right and left intersection points of the maxillary lateral border and a line passing through the nasal floor; interdental angle, defined as an angle between the right and left tooth axes determined by connecting the central fossa and palatal root apex; buccal bone thickness, defined as the distance from the buccal root surface to the outer border of the alveolar bone, along a horizontal line passing through the
Table 2. Comparison of skeletal and dentoalveolar measurements before (T1) and after (T2) expansion ( $\mathrm{n}=$ $14 ; \mathrm{mm})$

\begin{tabular}{lrrrc}
\hline & \multicolumn{1}{c}{ T1 } & \multicolumn{1}{c}{ T2 } & $\boldsymbol{\Delta}$ T2-T1 & $\boldsymbol{p}$-value \\
\hline IPMW & $39.2 \pm 3.1$ & $44.7 \pm 3.1$ & $5.5 \pm 1.4$ & $0.000^{\ddagger}$ \\
IMW & $50.2 \pm 3.6$ & $55.7 \pm 4.1$ & $5.4 \pm 1.7$ & $0.000^{\ddagger}$ \\
Z-Z & $124.9 \pm 3.5$ & $125.7 \pm 3.5$ & $0.8 \pm 0.5$ & $0.048^{*}$ \\
N-N & $23.8 \pm 1.8$ & $25.2 \pm 1.4$ & $1.4 \pm 1.0$ & $0.000^{\ddagger}$ \\
J-J & $65.0 \pm 4.4$ & $67.0 \pm 4.8$ & $2.0 \pm 1.4$ & $0.000^{\ddagger}$ \\
MA-MA & $62.3 \pm 4.8$ & $64.7 \pm 4.6$ & $2.4 \pm 1.3$ & $0.000^{\ddagger}$ \\
C6-C6 & $59.7 \pm 4.5$ & $62.9 \pm 4.4$ & $3.2 \pm 1.5$ & $0.000^{\ddagger}$ \\
Ag-Ag & $89.1 \pm 5.3$ & $89.0 \pm 4.9$ & $0.0 \pm 1.3$ & 0.947 \\
\hline
\end{tabular}

Data are presented as mean \pm standard deviation.

IPMW, Interpremolar width; IMW, intermolar width. Please refer to Table 1 for the definition of each landmark. Paired $t$-tests were performed according to the normality of the data; ${ }^{*} p<0.05,{ }^{*} p<0.001$.

furcation; and buccal alveolar height, defined as the distance from the buccal/mesiobuccal cusp tip to the buccal alveolar crest. Buccal bone thickness and alveolar height were measured on the right and left sides, and the mean values of the two measurements were used for statistical analyses.

\section{Statistical analysis}

The normality of data was determined using the Shapiro-Wilk test. Comparison of skeletal and dentoalveolar measurements before and after MARPE was performed using paired $t$-tests or Wilcoxon signed-rank tests, according to the normality of data distribution. Values of $p<0.05$ were considered statistically significant. All statistical analyses were performed using the SPSS version 15.0 software (SPSS Inc., Chicago, IL, USA). 
A single examiner performed all measurements. To determine the intraexaminer reliability, the same examiner reanalyzed seven randomly selected measurements within a 2-week interval. The resultant intraclass correlation coefficient (ICC) indicated high reliability $(\mathrm{ICC}>0.90)$.

\section{RESULTS}

The IPMW and IMW had increased by 5.5 and 5.4 $\mathrm{mm}$, respectively, after MARPE $(p<0.001$; Table 2). The midpalatal suture was separated, and the maxilla exhibited statistically significant lateral movement ( $p$ $<0.05$ ). The zygomatic arch and nasal cavity were widened by 0.8 and $1.4 \mathrm{~mm}$, respectively, and the lateral contour of the maxillary alveolus exhibited an expansion of 2.0-3.2 $\mathrm{mm}(p<0.001)$. The amount of expansion decreased with the superior positioning of anatomical structures, indicating a pyramidal pattern of maxillary expansion.

According to the findings of superimposition of the 3D skull models, all of the evaluated landmarks exhibited significant lateral movement ( $p<0.01$; Table 3$)$. The alare and ectocanine had shifted forward $(p<0.01)$, while the prosthion and ectomolare had shifted upward $(p<0.05)$. The distances between all of the right and left corresponding landmarks had increased after expansion $(p<0.01$; Table 4$)$.

Evaluation of coronal images of the first premolars and first molars demonstrated an increase in the nasal cavity width and basal bone width, buccal tipping of the maxillary first molars, and a decrease in the buccal bone thickness and alveolar bone height $(p<0.01$; Table 5). The increase in width of the basal bone was greater compared to that of the nasal cavity, which further confirmed the pyramidal pattern of maxillary expansion.

\section{DISCUSSION}

In the present study, we evaluated skeletal and dentoalveolar changes after MARPE in young adults with transverse maxillary discrepancy using CBCT. MARPE effectively achieved dentoalveolar as well as skeletal expansion by separation of the midpalatal suture. The

Table 3. Displacement in the maxilla, assessed in the transverse $(\mathrm{x})$, sagittal (y), and vertical (z) planes, after expansion (n $=28 ; \mathrm{mm}$ )

\begin{tabular}{|c|c|c|c|c|c|c|c|}
\hline & & Median & Minimum & Maximum & Range & IQR & $p$-value \\
\hline \multirow[t]{3}{*}{ Alare } & $\mathrm{x}$ & 0.8 & -1.3 & 2.4 & 3.6 & 1.1 & $0.000^{\ddagger}$ \\
\hline & $\mathrm{y}$ & -0.4 & -3.1 & 1.3 & 4.5 & 1.0 & $0.003^{*}$ \\
\hline & $\mathrm{z}$ & 0.02 & -1.7 & 1.6 & 3.3 & 1.4 & 0.799 \\
\hline \multirow[t]{3}{*}{ Ectocanine } & $\mathrm{x}$ & 0.8 & -0.6 & 3.7 & 4.3 & 1.5 & $0.000^{\ddagger}$ \\
\hline & $\mathrm{y}$ & -0.8 & -3.3 & 1.5 & 4.8 & 1.3 & $0.002^{*}$ \\
\hline & $\mathrm{z}$ & 0.4 & -1.7 & 3.0 & 4.7 & 1.5 & 0.280 \\
\hline \multirow[t]{3}{*}{ A-point } & $\mathrm{x}$ & 1.5 & 0.1 & 4.2 & 4.1 & 1.8 & $0.005^{*}$ \\
\hline & $\mathrm{y}$ & -0.4 & -1.9 & 2.0 & 3.9 & 1.5 & 0.174 \\
\hline & $\mathrm{z}$ & -0.3 & -2.5 & 2.3 & 4.8 & 1.8 & 0.264 \\
\hline \multirow[t]{3}{*}{ Prosthion } & $\mathrm{x}$ & 1.6 & -1.3 & 4.8 & 6.1 & 2.1 & 0.007 \\
\hline & $\mathrm{y}$ & 0.2 & -2.2 & 4.3 & 6.6 & 0.9 & 0.347 \\
\hline & $\mathrm{z}$ & 0.7 & -0.9 & 4.7 & 5.6 & 1.7 & $0.006^{*}$ \\
\hline \multirow[t]{3}{*}{ Ectomolare } & $\mathrm{x}$ & 1.3 & -0.9 & 3.6 & 4.5 & 1.6 & $0.003^{*}$ \\
\hline & $\mathrm{y}$ & -0.01 & -1.3 & 1.9 & 3.1 & 1.2 & 0.869 \\
\hline & $\mathrm{z}$ & -0.8 & -4.7 & 3.1 & 7.8 & 1.3 & 0.010 \\
\hline \multirow[t]{3}{*}{ Procesus zygomaticus } & $\mathrm{x}$ & 1.2 & -2.4 & 3.0 & 5.4 & 1.2 & $0.001^{\dagger}$ \\
\hline & $\mathrm{y}$ & 0.6 & -4.4 & 3.9 & 8.2 & 2.7 & 0.119 \\
\hline & $\mathrm{z}$ & 0.7 & -5.4 & 4.6 & 10.0 & 1.6 & 0.253 \\
\hline
\end{tabular}

IQR, Interquartile range.

Please refer to Table 1 for the definition of each landmark.

For the $\mathrm{x}$ coordinates, absolute values were used. For the $\mathrm{y}$ and $\mathrm{z}$ coordinates, positive values indicate backward and upward movements, respectively. Measurements were performed on both the right and left sides.

Comparison was performed using the Wilcoxon signed-rank nonparametric test; ${ }^{*} p<0.05,{ }^{\dagger} p<0.01,{ }^{*} p<0.001$. 
Park et al • Changes after MARPE in young adults

Table 4. Changes in the distances between the right and left corresponding landmarks after expansion $(n=14 ; m m)$

\begin{tabular}{lcccccc}
\hline & Median & Minimum & Maximum & Range & IQR & p-value \\
\hline Alare (1-2) & 1.4 & 0.1 & 3.3 & 3.3 & 1.7 & $0.001^{\dagger}$ \\
Ectocanine (3-4) & 2.5 & -0.5 & 4.4 & 4.8 & 3.1 & $0.003^{*}$ \\
A-point (5-6) & 3.0 & -0.5 & 6.8 & 7.2 & 4.0 & $0.003^{*}$ \\
Prosthion (7-8) & 3.5 & -0.5 & 6.6 & 7.1 & 4.7 & $0.006^{*}$ \\
Ectomolare (9-10) & 3.1 & -1.2 & 5.5 & 6.8 & 2.7 & $0.002^{*}$ \\
Procesus zygomaticus (11-12) & 2.8 & -3.0 & 5.5 & 8.5 & 3.5 & 0.009 \\
\hline
\end{tabular}

IQR, Interquartile range.

Please refer to Table 1 for the definition of each landmark.

Comparison was performed using the Wilcoxon signed-rank nonparametric tests; ${ }^{*} p<0.05,{ }^{\dagger} p<0.01$.

Table 5. Comparison of transverse dimensions before (T1) and after (T2) expansion measured on two-dimensional coronal images $(n=14)$

\begin{tabular}{llcccc}
\hline & & T1 & T2 & $\Delta$ T2-T1 & p-value \\
\hline Nasal cavity width $(\mathrm{mm})$ & PM1 & $24.5 \pm 3.2$ & $26.1 \pm 2.9$ & $1.6 \pm 1.8$ & $0.005^{\dagger}$ \\
& M1 & $29.5 \pm 2.6$ & $30.2 \pm 2.7$ & $0.7 \pm 0.9$ & $0.009^{\dagger}$ \\
Basal bone width $(\mathrm{mm})$ & PM1 & $40.1 \pm 8.1$ & $42.0 \pm 7.3$ & $1.9 \pm 2.3$ & $0.009^{\dagger}$ \\
& M1 & $64.5 \pm 5.5$ & $66.2 \pm 5.0$ & $1.7 \pm 1.8$ & $0.004^{\dagger}$ \\
Interdental angle $\left(^{\circ}\right)$ & PM1 & $0.4 \pm 11.4$ & $2.7 \pm 10.7$ & $2.2 \pm 10.6$ & 0.157 \\
Buccal bone thickness $(\mathrm{mm})$ & M1 & $40.4 \pm 8.9$ & $46.2 \pm 7.7$ & $5.8 \pm 5.7$ & $0.002^{\dagger}$ \\
& PM1 & $2.1 \pm 1.0$ & $1.0 \pm 1.0$ & $-1.1 \pm 0.8$ & $0.000^{\dagger}$ \\
Buccal alveolar height* $(\mathrm{mm})$ & M1 & $2.8 \pm 1.0$ & $2.3 \pm 1.3$ & $-0.6 \pm 1.0$ & $0.005^{\dagger}$ \\
& PM1 & $8.6 \pm 1.0$ & $10.7 \pm 3.1$ & $2.2 \pm 3.5$ & $0.006^{\dagger}$ \\
\hline
\end{tabular}

Data are presented as mean \pm standard deviation.

PM1, Maxillary first premolar; M1, maxillary first molar.

*Wilcoxon signed-rank nonparametric test was performed because buccal alveolar height was not normally distributed. Other variables were analyzed by paired $t$-tests; ${ }^{\dagger} p<0.01,{ }^{\dagger} p<0.001$.

increase in IMW width $(5.4 \mathrm{~mm})$ accounted for $37.0 \%$ of the skeletal expansion at the J-point $(2.0 \mathrm{~mm}), 22.2 \%$ of the alveolar expansion at the cementoenamel junction $(1.2 \mathrm{~mm})$, and $40.7 \%$ of the dental expansion at the cusp tip $(2.2 \mathrm{~mm})$. Despite the decrease in thickness and height of the buccal alveolus and the buccal tipping of the maxillary first molar, the changes observed after MARPE in the present study were similar to those observed after conventional RPE and can be considered clinically insignificant. ${ }^{8,20,21}$

Two-dimensional PA cephalograms and periapical or occlusal radiographs are considered adequate for ensuring the opening of the midpalatal suture. Apart from the increased radiation dose involved in CBCT, metal artifacts, voxel size, and superimposition errors may affect the spatial resolution of CBCT images, resulting in measurement errors. Nevertheless, the accuracy of CBCT has been well documented,,$^{13,17,22}$ and several reports on the assessment of conventional RPE outcomes using computed tomography or СBCT in growing patients have been published. ${ }^{14-16}$ In the present study, we evaluated CBCT data, the accuracy of which has been verified, ${ }^{13,17}$ to analyze the 3D movements of anatomical landmarks as well as the changes in the buccal alveolar bone, which is not possible with 2D modalities.

The bone-borne nature of the MARPE device can result in skeletal and alveolar expansion in young adults despite the increased resistance of the midpalatal and circumaxillary sutures from the age of 14-16 years. ${ }^{6,23}$ Among the 19 patients treated by MARPE in the present study, only 3 exhibited failure of opening of the midpalatal suture and were excluded, resulting in a success rate of $84.2 \%$. Skeletal expansion observed in the present study included the expansion of the zygomatic arch as well as nasal cavity. While the zygomatic arch expanded by less than $1 \mathrm{~mm}$, the expansion of the nasal cavity was more evident and 
would have resulted in increased air flow and improved nasal breathing. ${ }^{2,24}$ Thus, in the coronal plane, maxillary expansion followed a pyramidal pattern, similar to the expansion pattern reported with conventional RPE or SARPE. ${ }^{8,20,21}$

In the axial plane, however, the expansion did not demonstrate a pyramidal pattern, as previously reported. ${ }^{2,15}$ The amount of expansion was similar between the anterior and posterior regions at the crown (IPMW and IMW) and alveolar crest (prosthion, ectocanine, and ectomolare) levels. However, nasal cavity width in the premolar region had increased more obviously compared to that in the molar region, which may be attributed to the variations in nasal concha. Distribution of resistance from the maxilla might also cause parallel expansion. In similar age groups treated with SARPE, while parallel expansion of the maxilla was observed upon the release of pterygoid plates, ${ }^{1}$ the amount of anterior expansion was found to be greater than the amount of posterior expansion when only the pterygoid plates were not released. . $^{4,25}$

In the sagittal and vertical directions, MARPE did not result in clinically significant changes. On the 3D skull images, some of the landmarks exhibited forward or upward movements of less than $1 \mathrm{~mm}$. These movements might have resulted from changes in not only circumaxillary sutures, but also the spheno-occipital synchondrosis and orbital structures. ${ }^{26}$

The activation force of an RPE device initially results in the compression of the periodontal ligament, bending of the alveolar bone, and tipping of the anchored teeth. ${ }^{2}$ Therefore, a $1^{\circ}-24^{\circ}$-increase in molar inclination is inevitable, probably because of alveolar bending and/ or tipping of the posterior teeth. ${ }^{27}$ Previous studies regarding RPE and SARPE reported buccal tipping of $4.95^{\circ}-6.9^{\circ}$ of the maxillary first molar. ${ }^{15,28}$ These values are similar to those observed in the present study. The degree of buccal tipping of the first molar was greater compared to that of the first premolar. However, the first premolar exhibited similar or slightly greater increases in IMW, IPMW, nasal cavity, and basal bone widths than the first molar. Garib et al. ${ }^{21}$ also reported greater changes in the degree of molar inclination than that of premolar inclination. The higher density of the buccal cortical bone in the maxillary canine and premolar regions ${ }^{29}$ might have resulted in the greater buccal inclination of the first molar in comparison with that of the first premolar.

Tipping movements might cause changes in the alveolar bone. ${ }^{10,30} \ln$ the present study, we observed decreases in buccal plate thickness $(0.6-1.1 \mathrm{~mm})$ and buccal alveolar crest height (1.7-2.2 $\mathrm{mm})$ after MARPE. However, the buccal alveolar height might have been underestimated because of the tipping movements. In previous studies, RPE and SARPE were found to induce a similar decrease in buccal bone plate thickness. ${ }^{20,21}$ In one of the previous studies, ${ }^{20}$ the teeth appeared to have moved through the alveolus, resulting in decreased buccal bone thickness and increased lingual bone thickness. Tipping movements, which indicate greater lateral movement at the cervical level than at the apical level, along with the decrease in the alveolar bone thickness, might lead to the decrease in buccal alveolar crest height, which can eventually result in gingival recession. In children, the decrease in buccal alveolar crest height at the maxillary first molar after conventional RPE was reported to be approximately $1.23 \mathrm{~mm},{ }^{31}$ which is comparable to the corresponding value observed in the present study. Despite the lack of evidence regarding alveolar bone loss and subsequent gingival recession due to $\mathrm{RPE}^{32}$ the amount of marginal bone loss after RPE might be an indicator of future gingival recession, which calls for attention from the attending clinician.

The amount of appliance expansion $(6.7 \mathrm{~mm})$ was greater than the increase in the IPMW/IMW (5.5/5.4 $\mathrm{mm}$ ). This could indicate errors in counting of the number of appliance turns, because the amount of expansion was calculated on the basis of patient reports. Alternatively, it could indicate deformation of the appliance. Because of the controversies regarding nonsurgical expansion in adults, ${ }^{23}$ we were unable to address the effects of conventional RPE in patients with maxillary constriction. Additionally, because of ethical considerations, we could not include a control group of untreated patients in our study. Our study sample comprised only 14 young adults, which places emphasis on the necessity of further prospective studies involving larger numbers of patients and long-term evaluation of stability and periodontal adaptation after MARPE.

\section{CONCLUSION}

The null hypothesis was rejected. Within the limitations of this study, our results suggest the following.

- MARPE can be an effective treatment modality for the correction of maxillary transverse deficiency in young adults through separation of the midpalatal suture.

- Maxillary expansion achieved with MARPE exhibits a pyramidal pattern. In the present study, the degrees of skeletal, alveolar, and dental expansion were $37.0 \%, 22.2 \%$, and $40.7 \%$, respectively.

- Buccal tipping of maxillary teeth upon MARPE leads to the decrease in buccal alveolar bone thickness and crest height. Attending orthodontists should pay attention to these changes. 


\section{REFERENCES}

1. da Silva Filho OG, Montes LA, Torelly LF. Rapid maxillary expansion in the deciduous and mixed dentition evaluated through posteroanterior cephalometric analysis. Am J Orthod Dentofacial Orthop 1995;107:268-75.

2. Haas AJ. Palatal expansion: just the beginning of dentofacial orthopedics. Am J Orthod 1970;57:21955.

3. Shetty V, Caridad JM, Caputo AA, Chaconas SJ. Biomechanical rationale for surgical-orthodontic expansion of the adult maxilla. J Oral Maxillofac Surg 1994;52:742-9; discussion 750-1.

4. Asscherickx K, Govaerts E, Aerts J, Vande Vannet B. Maxillary changes with bone-borne surgically assisted rapid palatal expansion: A prospective study. Am J Orthod Dentofacial Orthop 2016;149:374-83.

5. Williams BJ, Currimbhoy S, Silva A, O'Ryan FS. Complications following surgically assisted rapid palatal expansion: a retrospective cohort study. J Oral Maxillofac Surg 2012;70:2394-402.

6. Persson M, Thilander B. Palatal suture closure in man from 15 to 35 years of age. Am J Orthod 1977;72:42-52.

7. Stuart DA, Wiltshire WA. Rapid palatal expansion in the young adult: time for a paradigm shift? J Can Dent Assoc 2003;69:374-7.

8. Rungcharassaeng K, Caruso JM, Kan JY, Kim J, Taylor G. Factors affecting buccal bone changes of maxillary posterior teeth after rapid maxillary expansion. Am J Orthod Dentofacial Orthop 2007;132:428.e1-8.

9. Capelozza Filho L, Cardoso Neto J, da Silva Filho OG, Ursi WJ. Non-surgically assisted rapid maxillary expansion in adults. Int J Adult Orthodon Orthognath Surg 1996;11:57-66; discussion 67-70.

10. Thilander B, Nyman S, Karring T, Magnusson 1. Bone regeneration in alveolar bone dehiscences related to orthodontic tooth movements. Eur J Orthod 1983;5:105-14.

11. Ramieri GA, Spada MC, Austa M, Bianchi SD, Berrone S. Transverse maxillary distraction with a bone-anchored appliance: dento-periodontal effects and clinical and radiological results. Int J Oral Maxillofac Surg 2005;34:357-63.

12. Lee KJ, Park YC, Park JY, Hwang WS. Miniscrewassisted nonsurgical palatal expansion before orthognathic surgery for a patient with severe mandibular prognathism. Am J Orthod Dentofacial Orthop 2010;137:830-9.

13. Mah JK, Danforth RA, Bumann A, Hatcher D. Radiation absorbed in maxillofacial imaging with a new dental computed tomography device. Oral
Surg Oral Med Oral Pathol Oral Radiol Endod 2003;96:508-13.

14. Akyalcin S, Schaefer JS, English JD, Stephens CR, Winkelmann S. A cone-beam computed tomography evaluation of buccal bone thickness following maxillary expansion. Imaging Sci Dent 2013;43:8590.

15. Christie KF, Boucher N, Chung CH. Effects of bonded rapid palatal expansion on the transverse dimensions of the maxilla: a cone-beam computed tomography study. Am J Orthod Dentofacial Orthop 2010;137(4 Suppl):S79-85.

16. Kartalian A, Gohl E, Adamian M, Enciso R. Conebeam computerized tomography evaluation of the maxillary dentoskeletal complex after rapid palatal expansion. Am J Orthod Dentofacial Orthop 2010;138:486-92.

17. Timock AM, Cook V, McDonald T, Leo MC, Crowe $\mathrm{J}$, Benninger BL, et al. Accuracy and reliability of buccal bone height and thickness measurements from cone-beam computed tomography imaging. Am J Orthod Dentofacial Orthop 2011;140:734-44.

18. Vanarsdall RL Jr. Transverse dimension and longterm stability. Semin Orthod 1999;5:171-80.

19. Magnusson A, Bjerklin K, Kim H, Nilsson P, Marcusson A. Three-dimensional assessment of transverse skeletal changes after surgically assisted rapid maxillary expansion and orthodontic treatment: a prospective computerized tomography study. Am J Orthod Dentofacial Orthop 2012;142: 825-33.

20. Corbridge JK, Campbell PM, Taylor R, Ceen RF, Buschang PH. Transverse dentoalveolar changes after slow maxillary expansion. Am J Orthod Dentofacial Orthop 2011;140:317-25.

21. Garib DG, Henriques JF, Janson G, de Freitas MR, Fernandes AY. Periodontal effects of rapid maxillary expansion with tooth-tissue-borne and tooth-borne expanders: a computed tomography evaluation. Am J Orthod Dentofacial Orthop 2006;129:749-58.

22. Jung PK, Lee GC, Moon CH. Comparison of conebeam computed tomography cephalometric measurements using a midsagittal projection and conventional two-dimensional cephalometric measurements. Korean J Orthod 2015;45:282-8.

23. Melsen B. Palatal growth studied on human autopsy material. A histologic microradiographic study. Am J Orthod 1975;68:42-54.

24. El H, Palomo JM. Three-dimensional evaluation of upper airway following rapid maxillary expansion: a CBCT study. Angle Orthod 2014;84:265-73.

25. Pinto PX, Mommaerts MY, Wreakes G, Jacobs WV. Immediate postexpansion changes following the use of the transpalatal distractor. J Oral Maxillofac Surg 
2001;59:994-1000; discussion 1001.

26. Bazargani F, Feldmann 1, Bondemark L. Threedimensional analysis of effects of rapid maxillary expansion on facial sutures and bones. Angle Orthod 2013;83:1074-82.

27. Lione R, Franchi L, Cozza P. Does rapid maxillary expansion induce adverse effects in growing subjects? Angle Orthod 2013;83:172-82.

28. Gurgel JA, Tiago CM, Normando D. Transverse changes after surgically assisted rapid palatal expansion. Int J Oral Maxillofac Surg 2014;43:31622.

29. Park HS, Lee YJ, Jeong SH, Kwon TG. Density of the alveolar and basal bones of the maxilla and the mandible. Am J Orthod Dentofacial Orthop
2008;133:30-7.

30. Tian YL, Liu F, Sun HJ, Lv P, Cao YM, Yu M, Yue Y. Alveolar bone thickness around maxillary central incisors of different inclination assessed with conebeam computed tomography. Korean J Orthod 2015;45:245-52.

31. Baysal A, Uysal T, Veli 1, Ozer T, Karadede 1, Hekimoglu S. Evaluation of alveolar bone loss following rapid maxillary expansion using conebeam computed tomography. Korean J Orthod 2013;43:83-95.

32. Handelman CS, Wang L, BeGole EA, Haas AJ. Nonsurgical rapid maxillary expansion in adults: report on 47 cases using the Haas expander. Angle Orthod 2000;70:129-44. 